\title{
BIORREFINARIA VIRTUAL: SIMULAÇÃO DINÂMICA DE EVAPORADORES
}

\author{
J. Ferreira, E. C. Biscaia Jr, A. R. Secchi \\ Universidade Federal do Rio de Janeiro, COPPE, Programa de Engenharia Química. \\ e-mail: mquagliata@peq.coppe.ufr..br
}

\begin{abstract}
RESUMO - As biorrefinarias produtoras de etanol a partir de cana-de-açúcar, na indústria nacional, são em sua maioria plantas que obtém o etanol a partir do processo de fermentação de primeira geração. Atualmente, está sendo estudada e avaliada tecnológica e economicamente, a produção de etanol de segunda geração. Uma das etapas de grande consumo energético comum aos processos de $1^{\mathrm{a}}$ e $2^{\mathrm{a}}$ geração é a etapa de concentração do caldo por sistemas de evaporadores. No presente trabalho é apresentada a modelagem fenomenológica em regime transiente de evaporador tipo Robert, implementada no simulador EMSO, com as propriedades físico-químicas calculadas pelo pacote termodinâmico VRTherm. A seção da calandra foi modelada como um trocador de calor do tipo casco e tubo com mudança de fase, seguida de um vaso de flash para separação das fases, com controle de nível e pressão. Foi utilizado um método de diferenças finitas na variável espacial para a resolução do sistema obtido de equações diferenciais parciais, e o uso do código DASSLC para a resolução do sistema de equações algébrico-diferenciais. As composições das fases líquida e vapor e as temperaturas obtidas estão em concordância com dados obtidos de unidades industriais e da literatura.
\end{abstract}

\section{INTRODUÇÃO}

O conceito de biorrefinaria é muito vasto; um deles é a conversão de biomassa em energia, combustível e/ou produtos químicos, onde a biomassa pode ser, por exemplo, canade-açúcar, beterraba, milho, madeira, grãos e algas. Existem várias classificações de biorrefinarias. Demirbas (2009) classifica as biorrefinarias segundo o processo de obtenção dos produtos: Bio-singas, pirólise, fermentação e processos hidrotérmicos. Outra classificação muito utilizada, também presente no trabalho de Demirbas (2009), é segundo o tipo de matéria-prima da biorrefinaria: $1^{\mathrm{a}}, 2^{\mathrm{a}}, 3^{\mathrm{a}}$, ou $4^{\mathrm{a}}$ geração. Nas plantas de $1^{\mathrm{a}}$ geração a matériaprima são sementes ou grãos, utilizando a celulose como composto intermédio; nas plantas de segunda geração são utilizados o bagaço, as palhas ou madeira, onde os produtos intermediários são a hemicelulose e a lignina; $3^{a}$ geração são as plantas onde se produzem 
combustíveis a partir de algas; e, por último, $4^{\mathrm{a}}$ geração são as plantas de produção de biodiesel a partir de óleos vegetais. Ainda é possível distinguir 4 tipos de tecnologias em uma refinaria: termoquímicos, bioquímicos, químicos e mecânicos/físicos.

O Brasil é o segundo maior produtor de etanol no mundo, as plantas industriais em funcionamento, atualmente, são plantas de primeira geração a partir de cana-de-açúcar, as quais utilizam um processo de fermentação como etapa para converter a sacarose presente no caldo de cana-de-açúcar em etanol. Portanto, o conhecimento de cada um dos processos presentes nas biorrefinarias de cana-de-açúcar é primordial para o avanço tecnológico e melhora de cada etapa.

A modelagem dinâmica de sistema de evaporação industrial tem sido pouco abordada na literatura, sendo um dos primeiros trabalhos realizado por Holland e Liapis (1983), apresentando a modelagem do evaporador tipo flash em estado não estacionário, para um sistema binário. Na simulação de sistemas de evaporadores de múltiplos efeitos na indústria sucroalcooleira, são destacados os trabalhos de Cadet et al. (1999) e Elhaq et al. (1999) que apresentaram a modelagem do sistema de evaporação para fins de controle; Manrique et al. (2011) que utilizaram a modelagem do evaporador tipo flash em estado não estacionário no software ECOSIMPRO; Jesus (2004) modelou evaporadores em estado estaconario e em transiente utilizando redes neuronais; e Silva (2013) que apresentou a modelagem e simulação dinâmica de evaporadores tipo flash e Robert aplicado a biorrefinarias.

No presente trabalho é abordada a modelagem fenomenológica dinâmica do evaporador tipo Robert no simulador EMSO, um simulador orientado por equações e por objetos, com as propriedades físico-químicas calculadas pelo pacote termodinâmico VRTherm.

\section{MODELAGEM DO EVAPORADOR TIPO ROBERT}

Para a modelagem do evaporador tipo Robert, foi considerado o evaporador em duas seções conectadas em serie, uma onde acontece a troca de calor nos tubos e outra seção onde acontece a separação das fases, modelada como um flash. Um esquema do sistema é apresentado na Figura 1.

\subsection{Modelagem da seção de flash}

A modelagem da parte que atua como flash é composta pelos balanços de massa por componentes e de energia. Considerando que as fases estão totalmente misturadas e que as perdas de energia pelas paredes do evaporador são desprezíveis, resulta nas seguintes equações:

$$
\begin{aligned}
& \frac{d n_{i}}{d t}=F_{o} z_{i}-F_{L} x_{i}-F_{V} y_{i} \quad \text { em que } n_{i}=N_{L} x_{i}+N_{V} y_{i}, \quad i=1, \ldots, N C \\
& \frac{d E}{d t}=F_{o} h_{o}-F_{L} h_{L}-F_{V} h_{V} \quad \text { em que } \quad E=N_{L} h_{L}+N_{V} h_{V}-P V
\end{aligned}
$$

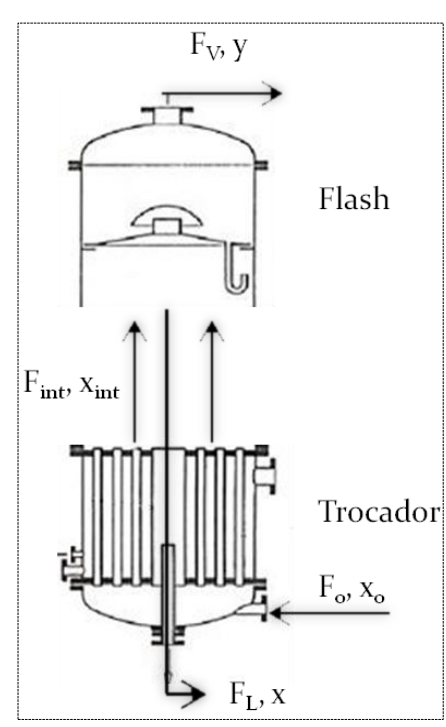

Figura 1 - Evaporador tipo Robert. 
Com: $\sum_{i=1}^{N C} x_{i}=1$ e $\sum_{i=1}^{N C} y_{i}=1$ e $N C$ o número de componentes.

Além dos balanços foram consideradas as equações de equilíbrio térmico, mecânico e químico:

$T_{L}=T_{V} \quad, \quad P_{L}=P_{V} \quad$ e $\quad \phi_{i}^{L} x_{i}=\phi_{i}^{V} y_{i}$

e foram adicionadas as equações dos controladores do tipo PI, para o controle de nível e de pressão, em que $u(t)$ representa as vazões de líquido, $F_{L}$, e vapor, $F_{V}$, respectivamente:

$u(t)=u_{b}+K_{c} e(t)+\frac{K_{c}}{\tau_{i}} \int_{0}^{t} e(\eta) d \eta \quad, u_{b}$ é o bias e $K_{c}$ e $\tau_{i}$ são parâmetros do controlador.

A seção de flash tem o volume constante e, portanto: $V=N_{L} v_{L}+N_{V} v_{V}$, em que $N_{L}$ e $N_{V}$ são as quantidades molares de líquido e vapor e $v_{L}$ e $v_{V}$ os volumes específicos molares.

Para o cálculo do nível considerou-se que o evaporador tem uma geometria cilíndrica. As propriedades termodinâmicas $\left(h_{L}, h_{V}, V_{L}, V_{V}, \phi_{L}, \phi_{V}\right)$ são funções da temperatura, pressão e composição da respectiva fase, e são calculadas a partir do plugin do EMSO, VRTherm.

\subsection{Modelagem da seção do trocador de calor}

No trocador de calor, o caldo está dentro dos tubos e o vapor (fluido aquecedor) do lado exterior dos tubos. Para o modelo do trocador de calor, foi considerado que não há acúmulo de massa dentro dos tubos, resultando na seguinte equação da continuidade, com $z$ a variável espacial, $\rho(z)$ a massa específica molar e $v(z)$ a velocidade do fluido:

$$
v(z) \rho(z)=v_{0} \rho_{0}
$$

Os balanços de massa para cada componente e de energia nos tubos são:

$$
\begin{aligned}
& \frac{\partial\left(\rho x_{i}\right)}{\partial t}=-\frac{\partial\left(v \rho x_{i}\right)}{\partial z} \\
& \frac{\partial(\rho h)}{\partial t}=-\frac{\partial(v \rho h)}{\partial z}+U \frac{4}{D_{t}}\left[T_{v a p}-T(z)\right]
\end{aligned}
$$

As propriedades termodinâmicas $(h, \rho$, ) são funções da temperatura, pressão e composição da mistura calculados através do plugin VRTherm do EMSO.

O coeficiente de transferência de calor, $U$, foi calculado a partir dos coeficientes de película interno e externo, $h_{\text {int }}$ e $h_{\text {ext }}$ obtidos a partir de correlações empíricas (Minton, 1986) em função dos números de Reynolds, Nusselt e Prandtl, assim como das propiedades do fluido interior e exterior aos tubos. 


\section{ALGORITMOS DE RESOLUÇÃO NUMÉRICA}

O EMSO tem vários algoritmos implementados para a resolução numérica de sistemas de equações diferenciais ordinárias ou sistemas de equações algébrico-diferenciais. Para a simulação do evaporador, foi utilizado o algoritmo DASSLC, que faz uso das fórmulas BDF e da técnica de predição-correção (Secchi, 2012).

Para a simulação primeiro foi realizada uma discretização do sistema de equações diferenciais parciais na variável $z$ pelo método de diferenças finitas de primeira ordem, gerando um sistema de equações algébrico-diferenciais ordinárias. Foram utilizados os valores de $1 \times 10^{-6}$ e $1 \times 10^{-8}$ como tolerância relativa e tolerância absoluta respectivamente, para o algoritmo DASSLC.

\section{RESULTADOS E DISCUSSÃO}

Para as simulações foi considerada como fluido de entrada uma mistura de dois componentes: água e sacarose, nas condições utilizadas na indústria.

Foram utilizadas as condições de operação e iniciais no evaporador indicadas pelas Tabelas 1 e 2.

Tabela 1 - Condições de operação

\begin{tabular}{|c|c|c|c|c|}
\hline Parâmetro & Valor (Unidades) & $\begin{array}{l}\text { Controlador } \\
\text { PID }\end{array}$ & Parâmetro & Valor (Unidades) \\
\hline$V$ & $153,74 \mathrm{~m}^{3}$ & \multirow{5}{*}{ pressão } & $u_{b}$ & 0,5 \\
\hline$D_{t}$ & $0,0331 \mathrm{~m}$ & & $K_{c}$ & 0,9 \\
\hline$N_{t}$ & 7575 & & $\tau_{i}$ & $40 \mathrm{~s}$ \\
\hline$H_{t}$ & $2,80 \mathrm{~m}$ & & Set Point & $1 \mathrm{~atm}$ \\
\hline$T_{\text {vap }}$ & $388,75 \mathrm{~K}$ & & & \\
\hline$F_{o}$ & $1380 \mathrm{kmol} / \mathrm{h}$ & \multirow{4}{*}{ nível } & $u_{b}$ & 0,5 \\
\hline$T_{o}$ & $358,15 \mathrm{~K}$ & & $K_{c}$ & 10 \\
\hline Po & $1,2 \mathrm{~atm}$ & & $\tau_{i}$ & $10 \mathrm{~min}$ \\
\hline$x_{O}$ & {$[0,99724 \quad 0,00276]$} & & Set Point & $2,9 \mathrm{~m}$ \\
\hline
\end{tabular}

Tabela 2 - Condições iniciais (Runyon, 1991)

\begin{tabular}{|l|c|}
\hline $\mathrm{x}(\mathrm{t}=0)$ & {$[0,996830,00317]$} \\
\hline Nivel $(\mathrm{t}=0)$ & $2,9 \mathrm{~m}$ \\
\hline $\mathrm{T}(\mathrm{t}=0)$ & $373 \mathrm{~K}$ \\
\hline
\end{tabular}


Por ser utilizado o método de diferenças finitas, foi encontrada a convergência da malha de discretização com 80 subdivisões, usando como critério a tolerância de 0,01 na pressão de saída do trocador de calor em qualquer tempo. Na Tabela 3 são apresentados os resultados dos testes de convergência da malha de discretização.

Tabela 3 - Convergência da malha de discretização e tempo de resolução

\begin{tabular}{|c|c|c|}
\hline Tamanho da malha & Módulo das diferenças de pressão & Tempo de resolução (s) \\
\hline 10 & & 2,836 \\
\hline 20 & 0,0148 & 3,395 \\
\hline 40 & 0,0121 & 6,606 \\
\hline 80 & 0,0099 & 10,729 \\
\hline
\end{tabular}

Na Figura 2 são apresentados os valores da pressão e da temperatura, no estado estacionário, no interior dos tubos ao longo do trocador de calor.
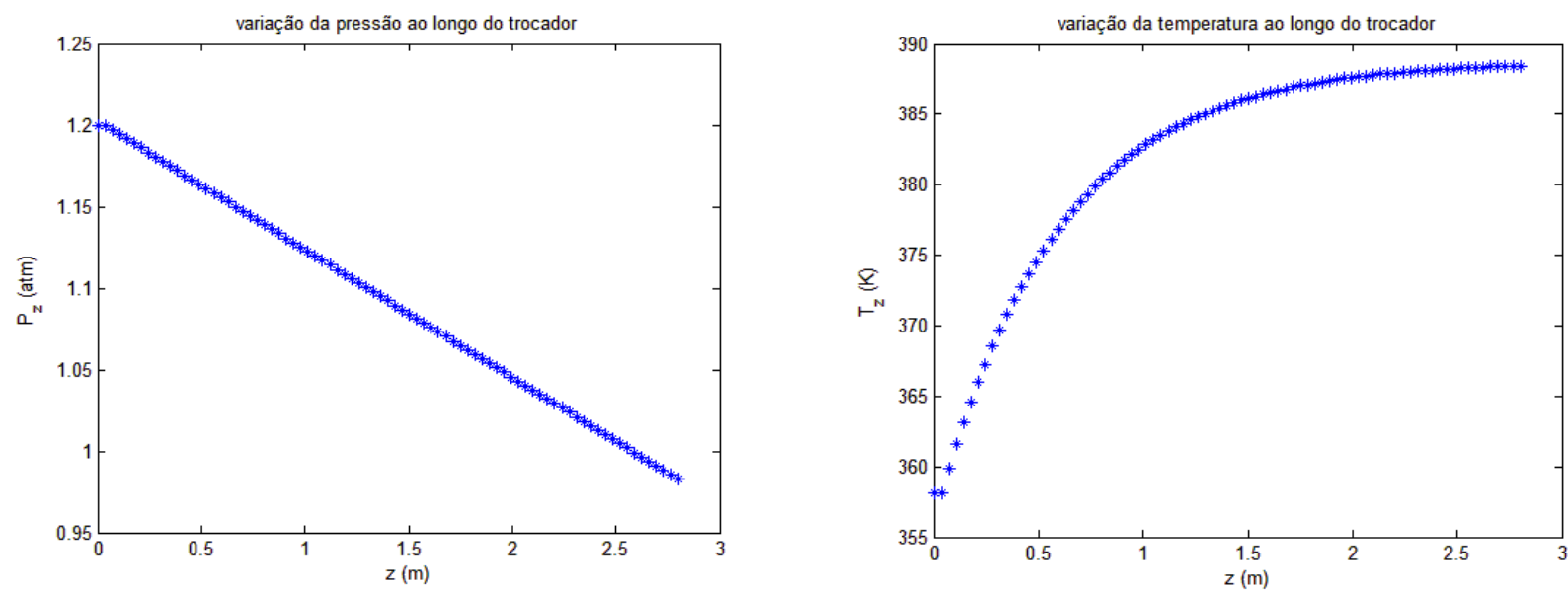

Figura 2 - Perfis estacionários de pressão e temperatura no trocador de calor.

Para a simulação do evaporador tipos flash, foi calculada uma taxa de calor transferido constante, de acordo com a taxa de calor transferido no estado estacionário para o evaporador tipo Robert.

De forma de poder comparar os dados obtidos com a literatura, foram realizadas a simulações, com um degrau de $+20 \%$ na concentração de entrada às 4 horas, da mesma forma que no trabalho de Runyon (1991).

$\mathrm{Na}$ Figura 3 são apresentados a concentração de sacarose na saída do liquido concentrado ao longo do tempo e o fluxo molar do vapor de saída para as simulações do evaporador tipo Robert e o evaporador tipo flash nas mesmas condições. A partir desta figura 
é possível observar que é atingido o estado estacionário para as duas simulações, e que as condições no estado estacionário atingido são similares.

É possível observar também a partir da Figura 3, que as trajetórias dinâmicas para chegar ao estado estacionário nos dois processos são diferentes. Por exemplo, a trajetória da concentração da sacarose para o modelo do evaporador tipo flash é bem mais lenta e não apresenta tempo morto quando comparada com a simulação do evaporador tipo Robert.

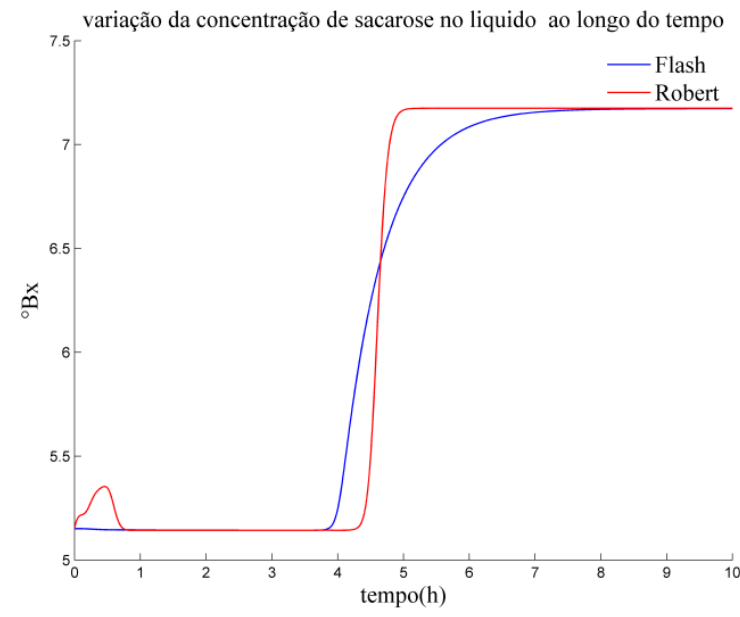

(a)

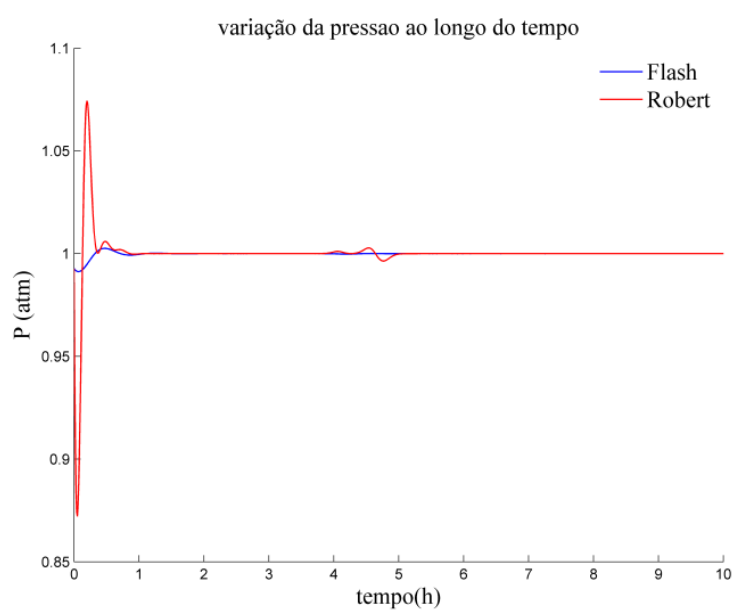

(c)

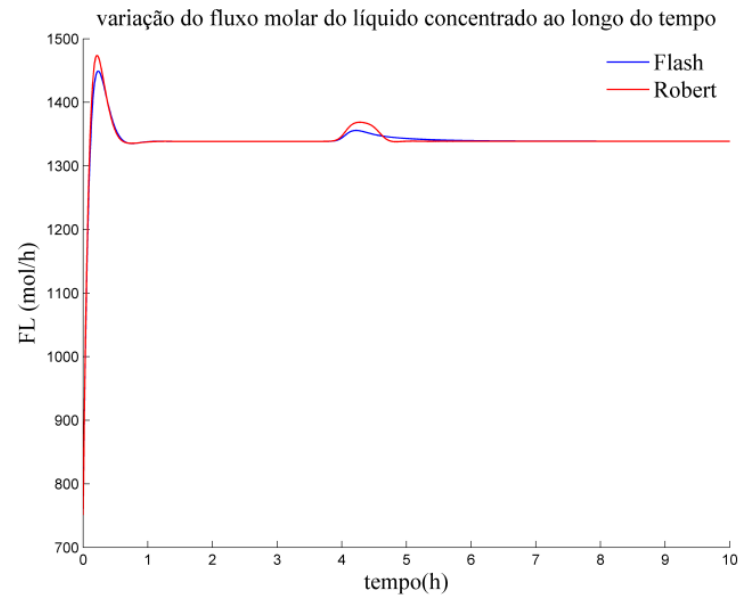

(b)

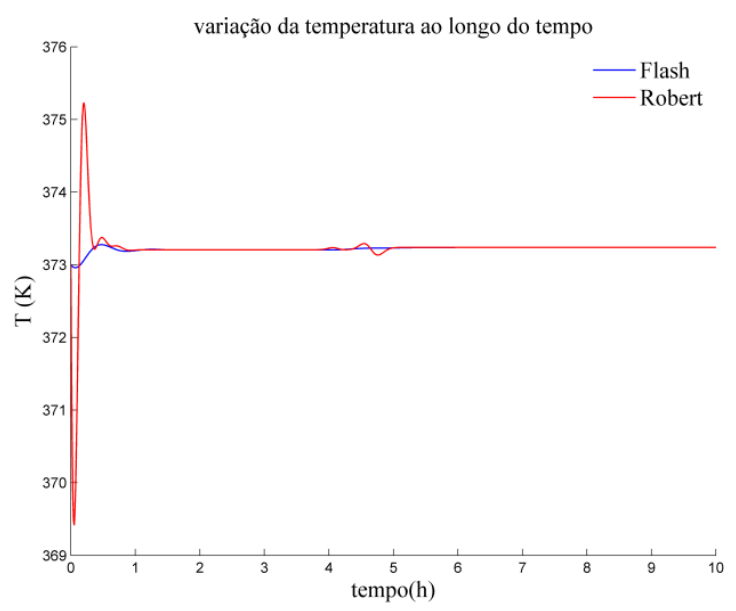

(d)

Figura 3 - Comparações dos modelos de evaporadores tipo Robert e tipo flash.

Foi achado que a resposta dos sistemas, Figura 3(a), a um degrau na concentração de entrada, são similares ao sistema de Runyon (1991) para o sistema sem controle de concentração de produto deste artigo. 


\section{CONCLUSÕES}

Foram construídos modelos matemáticos para os evaporadores tipo flash e Robert, onde a modelagem do evaporador do tipo Robert é apresentada em uma forma mais completa, tendo em conta as variações nas propriedades termodinâmicas ao longo dos tubos.

Os resultados obtidos nas simulações foram comparados, mostrando que os dois modelos alcançam o mesmo estado estacionário desde que a taxa de calor injetada no evaporador tipo flash seja a mesma do valor estacionário obtida no evaporador tipo Robert. Por outro lado, os comportamentos dinâmicos mostraram-se diferentes entre os dois modelos, com respostas mais lentas e sem tempo morto para o evaporador tipo flash.

Os valores de concentração, temperatura e pressão obtidos estão de acordo com os dados obtidos da literatura.

Ao ter os dois sistemas comportamentos dinâmicos diferentes, é de utilidade o conhecimento do tipo de comportamento dinâmico para ser usado, por exemplo, em atividades de partida da unidade ou projeto de sistemas de controle.

Com continuidade deste trabalho, pretende-se adicionar na mistura alimentada nos evaporadores outros componentes típicos da indústria sucroalcooleira.

\section{NOMENCLATURA}

\begin{tabular}{|c|c|c|c|}
\hline$e(t)$ & $\begin{array}{l}\text { erro, diferença entre o set point e a entrada } \\
\text { do sinal }\end{array}$ & $P$ & pressão no volume de controle, atm \\
\hline$E$ & Energia no volume de controle, $\mathrm{kJ}$ & $P_{L}$ & $\begin{array}{l}\text { pressão no volume de controle da fase } \\
\text { liquida, atm }\end{array}$ \\
\hline$F_{L}$ & fluxo molar da saída em fase liquida, $\mathrm{kmol} / \mathrm{h}$ & $P_{V}$ & $\begin{array}{l}\text { pressão no volume de controle da fase } \\
\text { vapor, atm }\end{array}$ \\
\hline$F_{o}$ & fluxo molar de entrada, $\mathrm{kmol} / \mathrm{h}$ & $T_{L}$ & $\begin{array}{l}\text { temperatura no volume de controle da } \\
\text { fase liquida, } \mathrm{K}\end{array}$ \\
\hline$F_{V}$ & fluxo molar da saída em fase vapor, $\mathrm{kmol} / \mathrm{h}$ & $T_{V}$ & $\begin{array}{l}\text { temperatura no volume de controle da } \\
\text { fase vapor, } \mathrm{K}\end{array}$ \\
\hline$h_{L}$ & $\begin{array}{l}\text { entalpia do fluido de saída em fase liquida, } \\
\mathrm{kJ} / \mathrm{kmol}\end{array}$ & $\rho$ & massa específica molar, $\mathrm{kmol} / \mathrm{m}^{3}$ \\
\hline$h_{o}$ & entalpia do fluido de entrada, $\mathrm{kJ} / \mathrm{kmol}$ & $U$ & $\begin{array}{l}\text { coeficiente de transferência de calor, } \\
\mathrm{kW} / \mathrm{h} / \mathrm{K} / \mathrm{m}^{2}\end{array}$ \\
\hline$h_{V}$ & $\begin{array}{l}\text { entalpia do fluido de saída em fase vapor, } \\
\mathrm{kJ} / \mathrm{kmol}\end{array}$ & $V$ & volume do evaporador, $\mathrm{m}^{3}$ \\
\hline$\tau_{i}$ & $\begin{array}{l}\text { constante do termo integral do controlador } \\
\text { (min) }\end{array}$ & $V_{L}$ & $\begin{array}{l}\text { volume molar da fase liquida no volume } \\
\text { de controle, } \mathrm{m}^{3} / \mathrm{kmol}\end{array}$ \\
\hline$K_{c}$ & $\begin{array}{l}\text { constante do termo proporcional do } \\
\text { controlador }\end{array}$ & $V_{V}$ & $\begin{array}{l}\text { volume molar da fase vapor no volume } \\
\text { de controle, } \mathrm{m}^{3} / \mathrm{kmol}\end{array}$ \\
\hline$n_{i}$ & $\begin{array}{l}\text { número de mols do componente } i \text { no volume } \\
\text { de controle, } \mathrm{kmol}\end{array}$ & $x_{i}$ & $\begin{array}{l}\text { fração molar do componente } i \text { na fase } \\
\text { liquida }\end{array}$ \\
\hline$N_{L}$ & $\begin{array}{l}\text { número de mols no volume de controle na } \\
\text { fase liquida, } \mathrm{kmol}\end{array}$ & $y_{i}$ & $\begin{array}{l}\text { fração molar do componente } i \text { na fase } \\
\text { vapor }\end{array}$ \\
\hline$N_{V}$ & $\begin{array}{l}\text { número de mols no volume de controle na } \\
\text { fase vapor, kmol }\end{array}$ & $Z_{i}$ & $\begin{array}{l}\text { fração molar do componente } i \text { no fluido } \\
\text { de entrada }\end{array}$ \\
\hline
\end{tabular}




\section{REFERÊNCIAS}

CADET, C., TOURÉ, Y., GILLES G., CHABRIAT, J.P., "Knowledge modeling and nonlinear predictive control of evaporators in cane sugar production plants", J. of Food Eng. 40, 59-70, 1999.

DEMIRBAS, M.F., "Biorefineries for biofuel upgrading: A critical review", App. Ener., v. 86, pp 151-161, 2009.

HOLLAND, C.D., LIAPIS, A.L., "Computer methods for solving dynamic separation problems", Estados Unidos: McGraw Hill Book Company, 1983.

JESUS, C. D. F., "Validação da simulação dinâmica das etapas de evaporação e cristalização da produção de açúcar com dados obtidos em plantas industriais", Tese de doutorado, UFSCar, 2004.

ELHAQ, L. S., GIRI, F., UNBEHAUEN, H., "Modelling identification and control of sugar evaporation theorical design and experimental evaluation", Con. Eng. Pra., vol. 7, pag. 931-942, 1999.

MANRIQUE, J., IPANIQUÉ, W., "Modelling and simulation of a multistage evaporator in ethanol plant using Ecosimpro environment", $9^{\text {th }}$ IEEE Int. Conf. on Cont. and Auto., 2011.

MINTON, P. E., "Handbook of evaporation technology", Estados Unidos: Noyes Publications, 1986.

RUNYON, C. H., RURNSEY, T. R., MCCARTHY, K. L., "Dynamic simulation of a nonlinear model of a double effect evaporator", J. Food Eng.,vol. 14, pag. 185-201, 1991.

SECCHI, A. R., "DASSLC User's manual v 3.8”, UFRGS, http://www.enq.ufrgs.br/enqlib/numeric/, 2012.

SILVA, P., "Simulação dinâmica e otimização de evaporadores de múltiplos efeitos em biorrefinarias", Dissertação de mestrado, UFRJ, 2013. 